\title{
Finite Element Technique for Optimal Pressure Recovery from Stream Function Formulation of Viscous Flows*
}

\author{
By M. E. Cayco and R. A. Nicolaides
}

\begin{abstract}
Following a general analysis of convergence for the finite element solution of the stream function formulation of the Navier-Stokes equation in bounded regions of the plane, an algorithm for pressure recovery is presented. This algorithm, which is easy to implement, is then analyzed and conditions ensuring optimality of the approximation are given. An application is made to a standard conforming cubic macroelement.
\end{abstract}

1. Introduction. The purpose of this paper is to provide a formulation and analysis of the stream function approach to the solution of the two-dimensional Navier-Stokes equations in polygonal simply connected domains. As with related approaches [2], [3], [4], [9], [10], [11], the pressure must be computed separately if it is required, and we give a natural algorithm for this purpose. In addition, we prove that the computed pressure will be "optimal" in the approximation-theoretic sense, for a particular kind of finite element space. Although specific numerical results are not given, they have been obtained by the authors and confirm the theoretical predictions.

For notation, let $\Omega$ be a bounded simply connected domain in $\mathbf{R}^{2} . L^{2}(\Omega)$ is the Hilbert space of square (Lebesgue) integrable functions with norm $\|\cdot\|_{0}$ and $L_{0}^{2}(\Omega)$ is the subspace of $L^{2}(\Omega)$ consisting of functions with zero mean. Let $H^{m}(\Omega)$ be the usual Sobolev space consisting of functions which together with their (distributional) derivatives up through order $m$ are in $L^{2}(\Omega)$. Denote the norm on $H^{m}(\Omega)$ by $\|\cdot\|_{m}$. Let $H_{0}^{m}(\Omega)$ be the completion of $C_{0}^{\infty}(\Omega)$ under the $\|\cdot\|_{m}$ norm. We equip $H_{0}^{m}(\Omega)$ with the seminorm $|\cdot|_{m}$, which is a norm equivalent to $\|\cdot\|_{m}$. Also, the dual of $H_{0}^{m}(\Omega)$ is denoted by $H^{-m}(\Omega)$, with norm $\|\cdot\|_{-m}$. Let $\vec{H}^{m}(\Omega),\left(\vec{H}_{0}^{m}(\Omega)\right)$ be the space $H^{m}(\Omega) \times H^{m}(\Omega)\left(H_{0}^{m}(\Omega) \times H_{0}^{m}(\Omega)\right)$ equipped with the following norm

$$
\|\vec{u}\|_{m}=\left(\left\|u_{1}\right\|_{m}^{2}+\left\|u_{2}\right\|_{m}^{2}\right)^{1 / 2} \quad\left(|\vec{u}|_{m}=\left(\left|u_{1}\right|_{m}^{2}+\left|u_{2}\right|_{m}^{2}\right)^{1 / 2}\right) \quad \text { where } \vec{u}=\left(\begin{array}{l}
u_{1} \\
u_{2}
\end{array}\right) \text {. }
$$

Denote the dual of $\vec{H}_{0}^{m}(\Omega)$ by $\vec{H}^{-m}(\Omega)$ with norm $\|\cdot\|_{-m}$. For each $\varphi \in H^{1}(\Omega)$, define

$$
\overrightarrow{\operatorname{curI}} \varphi=\left[\begin{array}{c}
\varphi_{y} \\
-\varphi_{x}
\end{array}\right]
$$

Received February 27, 1985; revised August 12, 1985.

1980 Mathematics Subject Classification. Primary 65N30.

* This work was supported by the Air Force Office of Scientific Research under Grant AFOSR-84-0137. 
2. Stream Function Formulation. A standard weak form for the stream function formulation for 2-dimensional stationary incompressible fluid flow (on a bounded simply connected domain) is

$$
\left\{\begin{array}{l}
\text { Find } \psi \in H_{0}^{2}(\Omega) \text { such that for all } \chi \in H_{0}^{2}(\Omega) \\
\nu \int_{\Omega} \Delta \psi \Delta \chi+\int_{\Omega} \Delta \psi\left(\psi_{y} \chi_{x}-\psi_{x} \chi_{y}\right)=\int_{\Omega} \vec{f} \cdot \overrightarrow{\operatorname{curl}} \chi
\end{array}\right.
$$

For brevity, we use the following notations:

$$
\begin{aligned}
a_{0}(\psi, \chi) & =\nu \int_{\Omega} \Delta \psi \Delta \chi \\
a_{1}(\zeta ; \psi, \chi) & =\int_{\Omega} \Delta \zeta\left(\psi_{y} \chi_{x}-\psi_{x} \chi_{y}\right), \\
l(\chi) & =\int_{\Omega} \vec{f} \cdot \overrightarrow{\operatorname{curl}} \chi
\end{aligned}
$$

Note that

$$
a_{0}(\psi, \chi) \leqslant \nu|\psi|_{2}|\chi|_{2} \text { for all } \psi, \chi \in H_{0}^{2}(\Omega)
$$

and that there exists a $\Gamma_{1}>0$ such that

$$
a_{1}(\zeta ; \psi, \chi) \leqslant \Gamma_{1}|\zeta|_{2}|\psi|_{2}|\chi|_{2} \text { for all } \zeta, \psi, \chi \in H_{0}^{2}(\Omega)
$$

and $\|l\|_{-2} \leqslant\|\vec{f}\|_{-1}$. The following theorem can be proved using the method of [8].

THEOREM 2.1. Let $\vec{f} \in \vec{H}^{-1}(\Omega)$ and define

$$
\nu^{*}=\left(\Gamma_{1}\|\vec{f}\|_{-1}\right)^{1 / 2} \text {. }
$$

Then for any $\nu>\nu^{*},(2.1)$ has a unique solution $\psi$. Moreover, $|\psi|_{2} \leqslant\|\vec{f}\|_{-1} / \nu$.

Let $V^{h} \subset H_{0}^{2}(\Omega)$ be a finite element trial space. Then to compute approximations to $\psi$, we solve for $\psi^{h} \in V^{h}$ in the following way:

$$
\left\{\begin{array}{l}
\text { Find } \psi^{h} \in V^{h} \text { such that for all } \chi^{h} \in V^{h} \\
a_{0}\left(\psi^{h}, \chi^{h}\right)+a_{1}\left(\psi^{h} ; \psi^{h}, \chi^{h}\right)=l\left(\chi^{h}\right)
\end{array}\right.
$$

Existence and uniqueness of the solution to (2.4) for $\nu>\nu^{*}$ follow from the fact that the properties of $a_{0}, a_{1}$ and $l$ used in the proof of Theorem 2.1 are inherited by any closed subspace of $H_{0}^{2}(\Omega)$. Moreover, $\left|\psi^{h}\right|_{2} \leqslant\|\vec{f}\|_{-1} / \nu$. We shall make use of the following estimate below. From here on, assume $\nu>\nu^{*}$.

THEOREM 2.2. Let $\psi$ be the solution to (2.1) and $\psi^{h}$ the solution to (2.4). Then

$$
\left|\psi-\psi^{h}\right|_{2} \leqslant c(\nu) \inf _{\chi^{h} \in V^{h}}\left|\psi-\chi^{h}\right|_{2}
$$

where $c(\nu)=\left(1+2 \Gamma_{1}\|\vec{f}\|_{-1} / \nu^{2}\right)\left(1-\Gamma_{1}\|\vec{f}\|_{-1} / \nu^{2}\right)^{-1} \leqslant c\left(\nu^{*}\right)$.

Proof. Since $V^{h} \subset H_{0}^{2}(\Omega)$, (2.1) holds for all $\chi^{h} \in V^{h}$. Subtracting (2.4) from (2.1) gives

$$
a_{0}\left(\psi-\psi^{h}, \chi^{h}\right)+a_{1}\left(\psi ; \psi, \chi^{h}\right)-a_{1}\left(\psi^{h} ; \psi^{h}, \chi^{h}\right)=0 \quad \text { for all } \chi^{h} \in V^{h}
$$


Observe that $a_{0}$ is bilinear and $a_{1}$ is trilinear. Also, $a_{1}(\zeta ; \psi, \chi)=-a_{1}(\zeta ; \chi, \psi)$ for all $\zeta, \psi, \chi \in H_{0}^{2}(\Omega)$. It soon follows that for any $\chi^{h} \in V^{h}$,

$$
\begin{aligned}
& a_{0}\left(\psi-\psi^{h}, \psi-\psi^{h}\right)+a_{1}\left(\psi^{h}-\psi ; \psi, \psi^{h}-\psi\right) \\
& \quad=a_{0}\left(\psi-\psi^{h}, \psi-\chi^{h}\right)+a_{1}\left(\psi^{h} ; \psi^{h}-\psi, \chi^{h}-\psi\right)+a_{1}\left(\psi^{h}-\psi, \psi, \chi^{h}-\psi\right) .
\end{aligned}
$$

Using (2.2), (2.3), and the fact that $a_{0}(\chi, \chi)=\nu|\chi|_{2}^{2}$ for all $\chi \in H_{0}^{2}(\Omega)$, we get

$$
\left(\nu-\Gamma_{1}|\psi|_{2}\right)\left|\psi-\psi^{h}\right|_{2}^{2} \leqslant\left(\nu+\Gamma_{1}|\psi|_{2}+\Gamma_{1}\left|\psi^{h}\right|_{2}\right)\left|\psi-\psi^{h}\right|_{2}\left|\psi-\chi^{h}\right|_{2} .
$$

Since $|\psi|_{2} \leqslant\|\vec{f}\|_{-1} / \nu$ and $\left|\psi^{h}\right|_{2} \leqslant\|\vec{f}\|_{-1} / \nu$, the conclusion is immediate.

Below, we shall provide an algorithm for pressure recovery and an error estimate for the particular space $V^{h} \subset H_{0}^{2}(\Omega)$ consisting of the Clough-Tocher triangles [6]. Let $\left\{\mathscr{Q}^{h}\right\}$ be a regular triangulation of $\Omega$. Each macrotriangle $Q \in \mathscr{Q}^{h}$ is divided into three subtriangles $Q_{1}, Q_{2}, Q_{3}$ by joining each vertex of $Q$ to its centroid. For this element, approximation theory shows [6]

$$
\left\{\begin{array}{l}
\text { for each } \chi \in H^{3}(\Omega) \cap H_{0}^{2}(\Omega), \text { there exists a } \chi^{h} \in V^{h} \text { such that } \\
\left|\chi-\chi^{h}\right|_{2} \leqslant c h^{2-s}\|\chi\|_{4-s}, \quad s=0,1,
\end{array}\right.
$$

where the lower value of $s$ assumes the implied extra regularity of $\chi$.

Although we do not make any further use of the following theorem, it is included here for completeness. Specifically, we can use a duality argument to get estimates for $\left|\psi-\psi^{h}\right|_{1}$ and $\left\|\psi-\psi^{h}\right\|_{0}$. Define the (linear) "dual" problem by:

$$
\left\{\begin{array}{l}
\text { Find } \zeta \in H_{0}^{2}(\Omega) \text { such that for all } \chi \in H_{0}^{2}(\Omega), \\
a_{0}(\chi, \zeta)+a_{1}(\psi ; \chi, \zeta)+a_{1}(\chi ; \psi, \zeta)=\langle g, \chi\rangle,
\end{array}\right.
$$

where $\psi$ is the solution to $(2.1)$ and $\langle\cdot, \cdot\rangle$ is the duality pairing in $L^{2}(\Omega)$. Since $\nu>\nu^{*},(2.8)$ is uniquely solvable for $g \in H^{-2}(\Omega)$. Moreover, $|\zeta|_{2} \leqslant c\|g\|_{-2}$.

TheOREM 2.3. Assume that $\psi \in H^{4}(\Omega) \cap H_{0}^{2}(\Omega)$. Moreover, assume that for each $g \in L^{2}(\Omega)$, the solution $\zeta$ to $(2.8)$ satisfies

$$
\zeta \in H^{4}(\Omega) \cap H_{0}^{2}(\Omega), \quad\|\zeta\|_{4} \leqslant c\|g\|_{-2} .
$$

Then there exist positive constants $C_{0}, C_{1}, C_{2}$ such that

$$
\begin{gathered}
\left|\psi-\psi^{h}\right|_{2} \leqslant C_{2} h^{2}, \\
\left|\psi-\psi^{h}\right|_{1} \leqslant C_{1} h^{3}, \\
\left\|\psi-\psi^{h}\right\|_{0} \leqslant C_{0} h^{4} .
\end{gathered}
$$

Proof. (2.9) follows immediately from (2.7) and Theorem 2.2. Let $\chi=\psi^{h}-\psi$ in (2.8), choose $\zeta^{h}$ corresponding to $\zeta$ as in (2.7), and use (2.6) to get

$$
\begin{aligned}
\left\langle g, \psi^{h}-\psi\right\rangle= & a_{0}\left(\psi^{h}-\psi, \zeta-\zeta^{h}\right)+a_{1}\left(\psi ; \psi^{h}-\psi, \zeta-\zeta^{h}\right) \\
& +a_{1}\left(\psi^{h}-\psi ; \psi^{h}, \zeta-\zeta^{h}\right)+a_{1}\left(\psi^{h}-\psi ; \psi-\psi^{h}, \zeta\right),
\end{aligned}
$$

hence

$$
\begin{aligned}
\left|\left\langle g, \psi^{h}-\psi\right\rangle\right| & \leqslant\left|\psi^{h}-\psi\right|_{2}\left|\zeta-\zeta^{h}\right|_{2}\left(\nu+\Gamma_{1}|\psi|_{2}+\Gamma_{1}\left|\psi^{h}\right|_{2}\right)+\Gamma_{1}|\zeta|_{2}\left|\psi-\psi^{h}\right|_{2}^{2} \\
& \leqslant K h^{4}\left(\|\zeta\|_{4}+|\zeta|_{2}\right) \leqslant K_{0} h^{4}\|g\|_{-2}, \\
& \leqslant K_{1} h^{4}\|g\|_{0} \quad \text { for } g \in L^{2}(\Omega) .
\end{aligned}
$$


Let $g=\Delta\left(\psi-\psi^{h}\right)$; then by $(2.12)$

$$
\left|\psi-\psi^{h}\right|_{1}^{2}=\left\langle g, \psi^{h}-\psi\right\rangle \leqslant K_{1} h^{4}\left|\psi^{h}-\psi\right|_{2} \leqslant K_{2} h^{6} \text { by (2.9), }
$$

hence

$$
\left|\psi-\psi^{h}\right|_{1} \leqslant C_{1} h^{3}
$$

Now set $g=\psi^{h}-\psi$. By (2.12) we get

$$
\left\|\psi-\psi^{h}\right\|_{0}^{2}=\left\langle g, \psi^{h}-\psi\right\rangle \leqslant K_{1} h^{4}\left\|\psi-\psi^{h}\right\|_{0},
$$

and (2.11) follows.

We make the remark that $(*)$ will hold if $\Omega$ is a polygon with maximum interior vertex angle $\theta<126^{\circ}$. See [4] for details.

3. Pressure recovery. We now turn to the important question of pressure recovery. Naturally, the momentum equations are used for this. Unlike other treatments, however, we shall avoid having to specify the pressure boundary conditions. The basic idea is to solve an equation of the form

$$
-\int_{\Omega} p \operatorname{div} \vec{v}=g(\vec{v})
$$

where $g$ is known. For convenience, let $b(\vec{v}, p)=-\int_{\Omega} p \operatorname{div} \vec{v}$. Note that $b$ is continuous and coercive on $\vec{H}_{0}^{1}(\Omega) \times L_{0}^{2}(\Omega)$ [8, Theorem 3.7, p. 35]. Thus it follows from Babuška's theorem [1] that for any $g \in\left[\vec{H}_{0}^{1}(\Omega)\right]^{\prime}$, a unique $p \in L_{0}^{2}(\Omega)$ exists such that the above equation holds for each $\vec{v} \in \vec{H}_{0}^{1}(\Omega)$. The term on the right-hand side depends on the solution $\psi$ of (2.1), the data $\vec{f}$ and $\nu$, and is given by

$$
\begin{aligned}
g(\vec{v}) & =g(\psi ; \vec{f}, \nu)(\vec{v}) \\
& =\langle\vec{f}, \vec{v}\rangle-\nu b_{0}(\overrightarrow{\operatorname{curI}} \psi, \vec{v})-b_{1}(\overrightarrow{\operatorname{curI}} \psi, \overrightarrow{\operatorname{curl}} \psi, \vec{v}),
\end{aligned}
$$

where $b_{0}(\vec{u}, \vec{v})=\int_{\Omega} \nabla \vec{u}: \nabla \vec{v}$ and $b_{1}(\vec{u}, \vec{w}, \vec{v})=\int_{\Omega}[(\vec{u} \cdot \nabla) \vec{w}] \cdot \vec{v}$. It follows directly from the continuity of $b_{0}$ and $b_{1}$ (see [8]) that

Lemma 3.1. For $\vec{f} \in \vec{H}^{-1}(\Omega), \psi \in H_{0}^{2}(\Omega), g$ as defined above is a bounded linear functional on $\vec{H}_{0}^{1}(\Omega)$. Moreover, for all $\psi, \chi \in H_{0}^{2}(\Omega), \vec{v} \in \vec{H}_{0}^{1}(\Omega)$,

$$
|g(\psi ; \vec{f}, \nu)(\vec{v})-g(\chi ; \vec{f}, \nu)(\vec{v})| \leqslant\left(\nu+\Gamma_{1}|\psi|_{2}+\Gamma_{1}|\chi|_{2}\right)|\psi-\chi|_{2}|\vec{v}|_{1} .
$$

Then we have

THEOREM 3.2. Given $\psi \in H_{0}^{2}(\Omega), \vec{f} \in \vec{H}^{-1}(\Omega)$, there exists a unique $p \in L_{0}^{2}(\Omega)$ such that

$$
b(\vec{v}, p)=g(\psi ; \vec{f}, \nu)(\vec{v}) \quad \text { for all } \vec{v} \in \vec{H}_{0}^{1}(\Omega) .
$$

It is the discretization of (3.2) which must be analyzed. The coercivity condition does not necessarily hold for arbitrary subspaces $X^{h} \subset \vec{H}_{0}^{1}(\Omega)$ and $S^{h} \subset L_{0}^{2}(\Omega)$. Also in discretizing (3.2), not only will $p$ and $\vec{v}$ be discretized, but so will $g$, i.e., $g(\psi ; \vec{f}, \nu)(\vec{v})$ has to be replaced by $g\left(\psi^{h} ; \vec{f}, \nu\right)\left(\vec{v}^{h}\right)$. Since the null space of $g\left(\psi^{h} ; f, \nu\right)$ does not necessarily coincide with the discretely div-free functions in $X^{h}$, the discretized analogue of (3.2) can only hold in some subspaces of $X^{h}$. This subspace 
is generally quite difficult to find. Hence, we introduce the following equivalent problem:

$$
\left\{\begin{array}{c}
\text { Find } \vec{w} \in \vec{H}_{0}^{1}(\Omega), p \in L_{0}^{2}(\Omega) \text { such that for all } \vec{v} \in \vec{H}_{0}^{1}(\Omega), q \in L_{0}^{2}(\Omega) \\
b_{0}(\vec{w}, \vec{v})+b(\vec{v}, p)=g(\psi ; \vec{f}, v)(\vec{v}) \\
b(\vec{w}, q)=0 .
\end{array}\right.
$$

(3.3) is uniquely solvable, and by Theorem $3.2, w=0$. We now discretize (3.3) as follows:

$$
\left\{\begin{array}{c}
\text { Find } \vec{w}^{h} \in X^{h}, p^{h} \in S^{h} \text { such that for all } \vec{v}^{h} \in X^{h}, q^{h} \in S^{h} \\
b_{0}\left(\vec{w}^{h}, \vec{v}^{h}\right)+b\left(\vec{v}^{h}, p^{h}\right)=g\left(\psi^{h} ; \vec{f}, \nu\right)\left(\vec{v}^{h}\right) \\
b\left(\vec{w}^{h}, q^{h}\right)=0
\end{array}\right.
$$

THEOREM 3.3. Let $\psi$ be the solution to (2.1) and $\psi^{h}$ the solution to (2.4). Let the test space $X^{h} \subset H_{0}^{1}(\Omega)$ and the trial space $S^{h} \subset L_{0}^{2}(\Omega)$ be chosen such that

$$
\left\{\begin{array}{l}
\text { for every } q^{h} \in S^{h}, \text { there exists } a \vec{v}^{h} \neq 0 \in X^{h} \text { such that } \\
b\left(\vec{v}^{h}, q^{h}\right) \geqslant \beta\left\|q^{h}\right\|_{0}\left|\vec{v}^{h}\right|_{1} \text { for some } \\
\text { positive constant } \beta, \text { independent of } q^{h}, \vec{v}^{h} .
\end{array}\right.
$$

Then (3.4) is uniquely solvable and

$$
\left\|p-p^{h}\right\|_{0} \leqslant c_{1} \inf _{q^{h} \in S^{h}}\left\|p-q^{h}\right\|_{0}+c_{2}\left|\psi-\psi^{h}\right|_{2}
$$

where $c_{1}, c_{2}$ are positive constants independent of $p, p^{h}, w^{h}, \psi$ and $\psi^{h}$.

Proof. It follows from primitive variable theory that (3.4) is uniquely solvable.

Subtracting (3.3) from (3.4), we get

$$
b_{0}\left(w^{h}, v^{h}\right)=g\left(\psi^{h} ; \vec{f}, \vec{v}\right)(\vec{v})-g(\psi ; \vec{f}, \nu)\left(\vec{v}^{h}\right)-b\left(\vec{v}^{h}, p^{h}-p\right) .
$$

Since $b\left(\vec{w}^{h}, p^{h}\right)=b\left(\vec{w}^{h}, q^{h}\right)=0$ for all $q^{h} \in S^{h}$,

where $K_{1}=\nu+2\|\vec{f}\| / \nu^{2}$.

$$
\left|w^{h}\right|_{1} \leqslant K_{1}\left|\psi-\psi^{h}\right|_{2}+\left\|p-q^{h}\right\|_{0}
$$

Hence, for any $\vec{v} \in X^{h}$,

$$
\begin{aligned}
& b\left(\vec{v}^{h}, q^{h}-p^{h}\right)=b\left(\vec{v}^{h}, q^{h}-p\right)+b\left(\vec{v}^{h}, p-p^{h}\right) \\
& \quad=b\left(\vec{v}^{h}, q^{h}-p\right)+b_{0}\left(w^{h}, v^{h}\right)+g(\psi ; \vec{f}, \nu)\left(\vec{v}^{h}\right)-g\left(\psi^{h} ; \vec{f}, \nu\right)\left(\vec{v}^{h}\right) \text { by }(*) .
\end{aligned}
$$

Using (3.5) we get

$$
\begin{aligned}
\left\|q^{h}-p^{h}\right\|_{0} & \leqslant \frac{1}{\beta}\left(\left\|q^{h}-p\right\|_{0}+\left|w^{h}\right|_{1}+K_{1}\left|\psi-\psi^{h}\right|_{2}\right) \\
& \leqslant \frac{1}{\beta}\left(2\left\|q^{h}-p\right\|_{0}+2 K_{1}\left|\psi-\psi^{h}\right|_{2}\right),
\end{aligned}
$$

hence

$$
\begin{aligned}
\left\|p-p^{h}\right\|_{0} & \leqslant\left\|p-q^{h}\right\|_{0}+\left\|q^{h}-p^{h}\right\|_{0} \\
& \leqslant c_{1} \inf _{q^{h} \in S^{h}}\left\|p-q^{h}\right\|_{0}+c_{2}\left|\psi-\psi^{h}\right|_{2},
\end{aligned}
$$

where $c_{1}=1+2 / \beta, c_{2}=2 K_{1} / \beta$. 
Let $\left\{\mathscr{Q}^{h}\right\}$ be a uniformly regular family of triangulations of $\Omega$. For each $2^{h}$, associate a triangulation $\mathscr{T}^{h}$ of $\Omega$ consisting of subtriangles constructed as in Section 2. Let

$$
\begin{aligned}
& X^{h}=\left\{\vec{v}^{h} \in \vec{H}_{0}^{1}(\Omega) \mid \vec{v}^{h} \text { is a continuous piecewise quadratic on } \mathscr{T}^{h}\right\} \\
& S^{h}=\left\{q^{h} \in L_{0}^{2}(\Omega) \mid q^{h} \text { is a piecewise linear function on } \mathscr{Q}^{h}\right\} .
\end{aligned}
$$

To get convergence, it is sufficient to show that the family $\left\{X^{h}, S^{h}\right\}$ is div-stable, i.e., for all $X^{h}, S^{h}$ in the family, (3.5) holds with $\beta$ independent of $h$. This can be done using the local test of [5], since the inclusion $S^{h} \supset$ \{piecewise constants on $\left.2^{h}\right\}$ is selfevident. Thus, it suffices to show that $\left\{X^{h}, S^{h}\right\}$ is locally div-stable, i.e., there exists a $\hat{c} \neq \hat{c}(h)$ such that for all $Q \in \mathscr{Q}^{h}$, and for all $q^{h} \in S^{h} \cap L_{0}^{2}(Q)$, there exists a $\vec{v}^{h} \in X^{h} \cap H_{0}^{1}(Q)$ such that

$$
\int_{Q}\left|\nabla \vec{v}^{h}\right|^{2} \leqslant \hat{c} \int_{Q}\left(q^{h}\right)^{2} \text { and } \int_{Q} q^{h} \operatorname{div} \vec{v}^{h} \geqslant \int_{Q}\left(q^{h}\right)^{2}
$$

THEOREM 3.4. $\left\{X^{h}, S^{h}\right\}$ is locally div-stable.

Proof. We shall use the following inequalities:

$$
\begin{gathered}
\int_{T}\left|\nabla \vec{v}^{h}\right|^{2} \leqslant C_{1} h^{-2} \int_{T}\left|\vec{v}^{h}\right|^{2} \quad \text { for all } T \in \mathscr{T}^{h}, \vec{v}^{h} \in X^{h}, \\
\int_{Q}\left|\nabla q^{h}\right|^{2} \leqslant C_{1} h^{-2} \int_{Q}\left(q^{h}\right)^{2} \quad \text { for all } Q \in \mathscr{Q}^{h}, q^{h} \in S^{h}, \\
\int_{Q}\left(q^{h}\right)^{2} \leqslant C_{2} h^{2} \int_{Q}\left|\nabla q^{h}\right|^{2} \quad \text { for all } Q \in \mathscr{Q}^{h}, q^{h} \in S^{h} \cap L_{0}^{2}(Q),
\end{gathered}
$$

where $C_{1}$ and $C_{2}$ are positive constants independent of $h$.

Now, let $Q \in \mathscr{Q}^{h}$ and $q^{h} \in S^{h} \cap L_{0}^{2}(Q)$ be given. Let $\lambda_{i}, i=1,2,3$, be the barycentric coordinates associated with $Q$, and let $Q_{1}, Q_{2}, Q_{3} \in \mathscr{T}^{h}$ be such that $\cup_{i=1}^{3} Q_{i}=Q$ and one edge of $Q_{i}$ lies on $\lambda_{i}=0, i=1,2,3$. Define $P: \Omega \rightarrow \mathbf{R}$ by

$$
P= \begin{cases}\left(3 \lambda_{i}\right)^{2} & \text { on } Q_{i}, \\ 0 & \text { on } \Omega \backslash Q .\end{cases}
$$

Then, a direct calculation shows that for all $\alpha, \beta \in \mathbf{R}$,

$$
\left(\begin{array}{l}
\alpha P \\
\beta P
\end{array}\right) \in \vec{H}_{0}^{1}(Q) \cap X^{h}, \quad \int_{Q} P=\frac{1}{6} \text { area } Q, \quad \int_{Q} P^{2}=\frac{1}{15} \text { area } Q .
$$

Define $\vec{v}^{h}=-6 C_{2} h^{2} \nabla q^{h} P$, where $C_{2}$ is given by (3.11) and $P$ is defined by (3.12). Since $\nabla q^{h}$ is constant on $Q$ and $P \in H_{0}^{1}(Q)$, it follows that $\vec{v}^{h} \in X^{h} \cap \vec{H}_{0}^{1}(Q)$. Moreover,

$$
\begin{aligned}
\int_{Q} q^{h} \operatorname{div} \vec{v}^{h} & =-\int_{Q}\left(\nabla q^{h}\right) \cdot \vec{v}^{h}=6 C_{2} h^{2}\left|\nabla q^{h}\right|^{2} \int_{Q} P \\
& =C_{2} h^{2}\left|\nabla q^{h}\right|^{2} \text { area } Q \geqslant \int_{Q}\left(q^{h}\right)^{2}
\end{aligned}
$$


Also,

$$
\begin{aligned}
\int_{Q}\left|\nabla \vec{v}^{h}\right|^{2} & \leqslant C_{1} h^{-2} \int_{Q}\left|\vec{v}^{h}\right|^{2}=36 C_{1} C_{2}^{2} h^{2}\left|\nabla q^{h}\right|^{2} \int_{Q} P^{2} \\
& =\frac{36}{15} C_{1} C_{2}^{2} h^{2}\left|\nabla q^{h}\right|^{2} \text { area } Q \leqslant \frac{36}{15} C_{1}^{2} C_{2}^{2} \int_{Q}\left(q^{h}\right)^{2} .
\end{aligned}
$$

Thus, the local stability is proved, and so by [5] global stability in the sense of (3.5) follows.

Acknowledgment. We would like to thank Dr. James M. Boland for the helpful discussions related to this work.

Department of Mathematics

Carnegie-Mellon University

Pittsburgh, Pennsylvania 15213

1. I. BABUSKA \& A. K. Azız, The Mathematical Foundations of the Finite Element Method with Applications to Partial Differential Equations, Academic Press, New York, 1972.

2. A. S. Benjamin \& V. E. Denny, "On the convergence of numerical solutions for 2-D flows in a cavity at large Re,” J. Comput. Phys., v. 33, 1979, pp. 340-358.

3. P. L. Betts \& V. Haroutunian, “A stream function finite element solution for two-dimensional natural convection," Finite Element Flow Analysis (T. Kawai, ed.), Univ. of Tokyo Press, 1982, pp. 279-288.

4. H. BLum \& R. RANNACHER, "On the boundary value problem of the biharmonic operator on domains with angular corners," Math. Methods Appl. Sci., v. 2, 1980, pp. 556-581.

5. J. M. Boland \& R. A. Nicolaides, "Stability of finite elements under divergence constraints," SIAM J. Numer. Anal., v. 20, no. 4, 1983, pp. 722-731.

6. P. G. Ciarlet, The Finite Element Method for Elliptic Problems, North-Holland, Amsterdam, 1980.

7. U. Ghia, K. N. Ghia \& C. T. Shin, "High Re solutions for incompressible flow using the Navier-Stokes equations and a multigrid method," J. Comput. Phys., v. 48, 1982, pp. 387-411.

8. V. Girault \& P.- A. Raviart, Finite Element Approximations of the Navier-Stokes Equations, Lecture Notes in Math., vol. 749, Springer-Verlag, New York, 1979.

9. M. D. Olson \& S.- Y. TUANN, “New finite element results for the square cavity," Comput.\& Fluids, v. 7, 1979, pp. 123-135.

10. M. D. Olson \& S.- Y. TUANN, "Review of computing methods for recirculating flows," J. Comput. Phys., v. 29, 1978, pp. 1-19.

11. R. SChreiber \& H. B. Keller, "Driven cavity flows by efficient numerical techniques," J. Comput. Phys., v. 49, 1983, pp. 310-333. 